\title{
Dose Length Product and Computed Tomography Dose Index as Parameters for Patients Doses
}

\author{
H. Osman ${ }^{*}$, Aseel Alotaibi, Nawaf Alotaibi, Fahad Alotaibi, Mohannad Alqhtani, Abdulraman Alsalmi, Faisal Alkhaily
}

Taif University College of Applied Medical Science, King of Saudi Arabia

DOI: $10.36347 /$ sjams.2020.v08i05.023

| Received: 15.04.2020 | Accepted: 27.04.2020 | Published: 16.05.2020

*Corresponding author: H. Osman

\section{Abstract}

Original Research Article

Background: The study Assess the measurement of radiation doses indicators during abdominal CT investigation by computed tomography, these examinations irradiate the patients with a relatively high doses so the study aimed to measure these indicators and then compared the results with different literature. Materials and methods: The radiation doses indicators were measured for 29 patients at King Abdul Aziz Specialist Hospital in Taif .The bio-data (height, weight and body mass index BMI) was recorded, computed tomography dose index (CTDI) and dose length product (DLP) have been used to indicate the radiation dose, which were displayed on computed tomography machine made by Siemens. Microsoft Excel program was used to analyze the data, in turn plotted into graphs. Main results: The average CTDIs, DLPs and body mass index BMI was $13.56 \pm 3,98,538,3$ mGy per centimeter $\pm 193,4$ and 24.2 $\mathrm{Kg} / \mathrm{m} 2 \pm 4.8$ respectively. Also study revealed that there was correlation between CTDIs and DLPs, also correlation between patient body mass Index and DLP was found, No a correlation between the time and DLPs or even between ages and CTDI were found. Recommendations: The study recommended more studies in this field specifically for computed tomography angiogram CTA.

Keywords: Computed tomography, Abdomen, DLP, CTDIs.

Copyright @ 2020: This is an open-access article distributed under the terms of the Creative Commons Attribution license which permits unrestricted use, distribution, and reproduction in any medium for non-commercial use (NonCommercial, or CC-BY-NC) provided the original author and source are credited.

\section{INTRODUCTION \\ Background}

Computed tomography (CT), was originally known as "EMI scan" because it was developed at a research branch of Electric and Musical Industries in England. It was later known as computed axial tomography (CAT scan)[1].

CT produces multiple images of data in the form of a slab or a block of the body that is sliced and stacked like a sliced loaf of bread. Any slice, of any desired thickness, from anywhere out of the "loaf" can be taken out of the slab or "loaf" to be viewed and manipulated through a process known as windowing, in order to see various structures based on shades of gray, black and white on a set numbered scale .CT is a special $\mathrm{x}$-ray technique; in this system, the patient lies on a bed while the $\mathrm{x}$-ray tube and the detector system are taking different tomograms of him or her. The way this is achieved depends on the scanner itself .basic principle of Computed Tomography is that it takes different radiographs of the body at different angles. Each one of these radiographs is called a projection. The $\mathrm{x}$-ray tube is placed in front of a detector system, and the patient lies on a bed between the x-ray tube and the detector system. An initial radiograph is taken, and then more radiographs are taken at different angles. This is achieved with the rotation of the x-ray tube and the detector system. Then, the detector system sends the data to a computer. The data is stored and processed, to display the final image of the body section.

CT of abdomen is superior at visualizing the liver, spleen, pancreas and other internal organs. CT of abdomen can be done using contrast or without it, contrast may be: orally drinken to fill the stomach, small bowel and colon, or intravenously IV contrast, through injection IV, to show internal organs like the liver, spleen, pancreas and kidneys [2].

The use of contrast in abdomen CT depends on the need, the symptos and if you are able to have it. Some abdomen scans can be performed with oral only; some with IV only and some with both oral and IV contrast are often necessary. Ct of abdomen, especially in trauma situations like a car accident, is performed with IV contrast in order to visualize internal organs for tear or assess injury. Visualization of the pancreas is best seen using CT with IV and oral contrast to evaluate the extent of conditions such as pancreatitis, pancreatic 
cysts and lesions or tumors of the pancreas. Drinking oral contrast outlines the head of the pancreas as the stomach empties itself in a "C-shaped" loop around the pancreatic head [3].

\section{Importance of the study}

The radiation dose of $\mathrm{CT}$ is relatively high compared to other imaging modalities, the most cases presented in the departments under investigation was abdominal CT scan, so researchers motivated for this research in order to propose protocol to reduce the radiation dose and establish local diagnostic reference level.

\section{Study motivations}

In this study, the optimization potential on abdomen/pelvic examination has been examined. This examination covers a large volume of the patient containing several vital and radiation-sensitive organs. Generally reduced patient dose, while maintaining an acceptable image quality for this specific protocol may have a significant impact on this patient group at intended Hospitals There was a request from the management at the radiological department on optimizing this particular procedure as many patients undergo this examination.

The noise increase at lower doses and lowcontrast object detection is reduced with increasing noise (Curry, Dowdey et al. 1990) In the abdomen area the most significant challenge related to lowering the dose is the loss of low-contrast object detectability.

\section{Objectives}

The main objective of this thesis were to measure radiation dose during $\mathrm{CT}$ examination for abdomen

\section{Materials ANd Methods}

Twenty nine patients divided according to gender as follow: Eight females and Twenty-two males, they all requested for Computed tomography for abdomen or pelvis, with different bio-data and body characteristic.. The study was conducted at King Abdul Aziz Specialist Hospital and King Fiasal hospital in Taif. 64 slices, computed tomography machine, made in Germany, by Siemens and was installed In $1435 \mathrm{H}$. the machine was shown in figure 1 , Gantry and table.

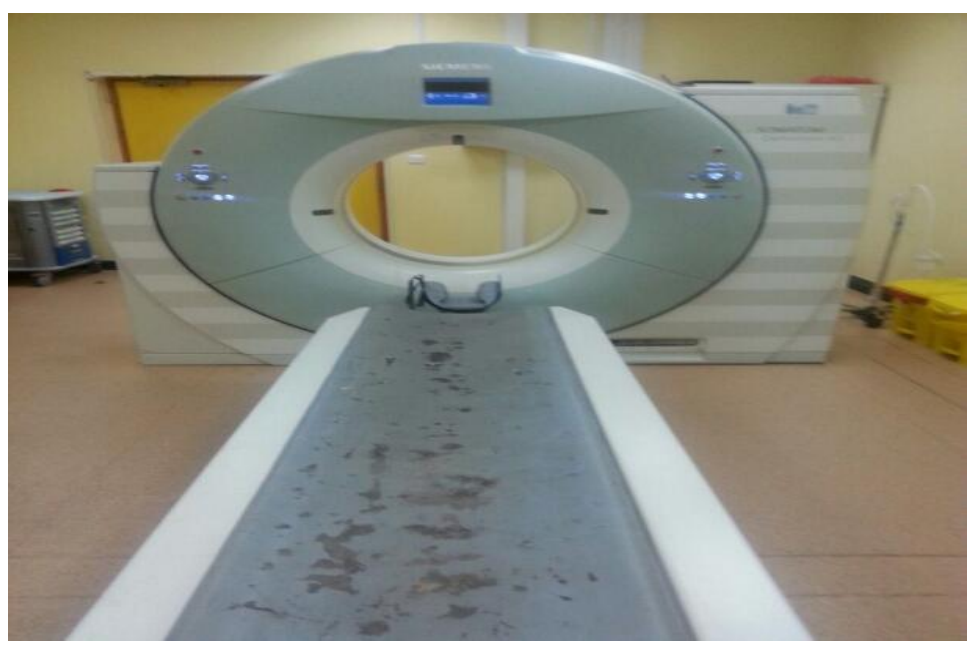

Fig-1: Layout of machine used during the study

The survey dyration was from January till March 2020. Data collection sheet was designed to collect data from the above aforementioned hospitals. Special data collection sheet was filled by researchers according to type of examinations required and patient fellow. all required data for this type of study were written in the sheet just like age, gender CT dose Index and $\mathrm{Ct}$ dose volume, some data were patient related and some other data were machine protocol related. Data which was machine dependent were calculated with the CT machine, like CT dose index or CT dose volume and slice thickness.

Samples were collected from the hospital, King Abdul Aziz Specialist hospital (KAASH) in Taif city. All patient referred for the department for abdomen CT during the study period. Patient referred out of working day hour were not included.

Patient referred for $\mathrm{Ct}$ during the study period but not for CT abdomen, for other CT investigation, just like chest skull or extremities.

Microsoft Excel 2007 was used to analyze data collected from the hospitals. After data entry the average and standard deviation was calculated for most variables. Also curve and figures were plotted to facilitate reading the results. 


\section{RESULTS}

Table-1: population characteristic, effective mAs, average dose length product DLP and CT dose Index CTDI

\begin{tabular}{|c|c|c|c|c|}
\hline Parameter & Average & Max Min & Min & deviation \pm STD \\
\hline Patient height (cm) & 151.4 & 172 & 138 & 14.7 \\
\hline Patient weight Kg & 76.7 & 103 & 55 & 23.64 \\
\hline Patient BMI Kg/l2 & 25.8 & 31.3 & 24.2 & 4.8 \\
\hline Patient age (year) & 51.10 & 84 & 17 & 21.3 \\
\hline DLP mGy.cm & 538.3 & 955 & 274.2 & 193.4 \\
\hline CTDI & 13.56 & 22.04 & 6.86 & 3.98 \\
\hline Effective mAs & 203.10 & 380 & 80 & 64.71 \\
\hline Time (sec) & 10.36 & 10.36 & 2.7 & 1.93 \\
\hline
\end{tabular}

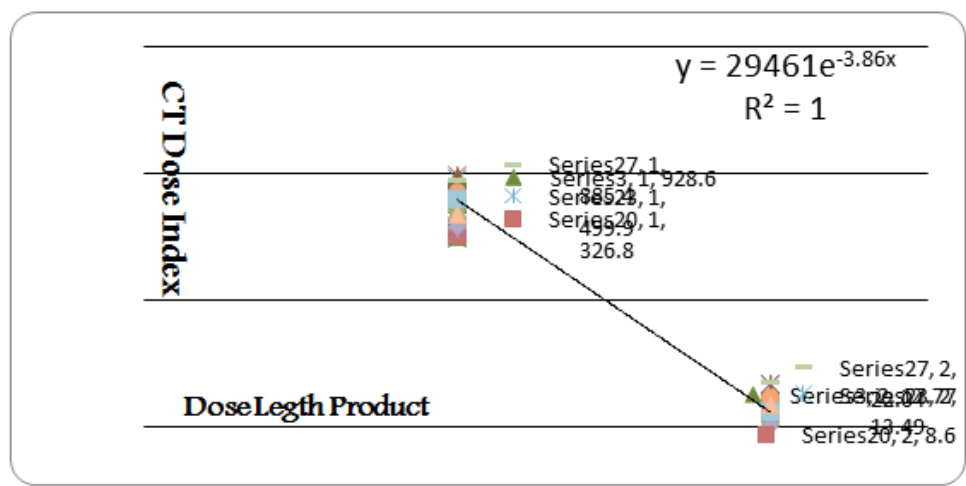

Fig-2: Correlation of dose DLP and CTDI

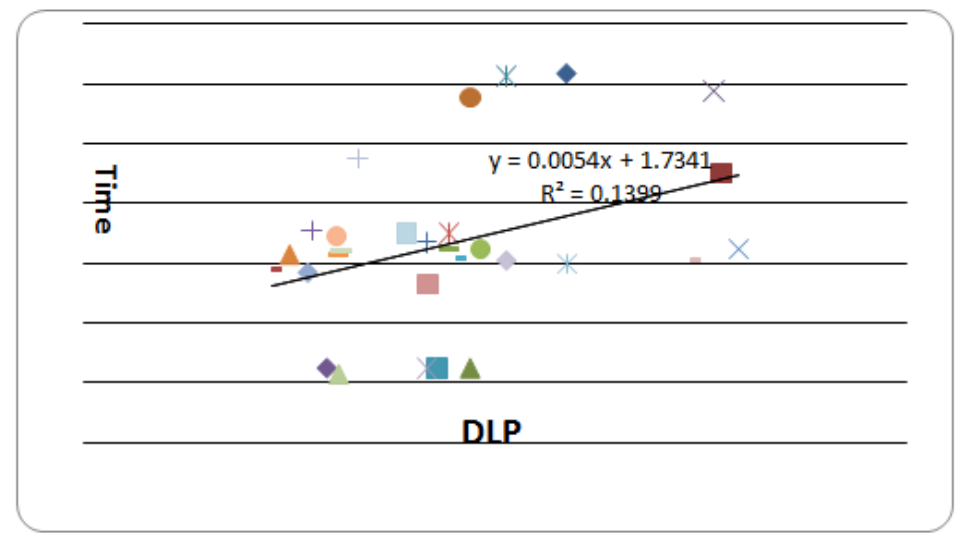

Fig-3: Dose length product and time

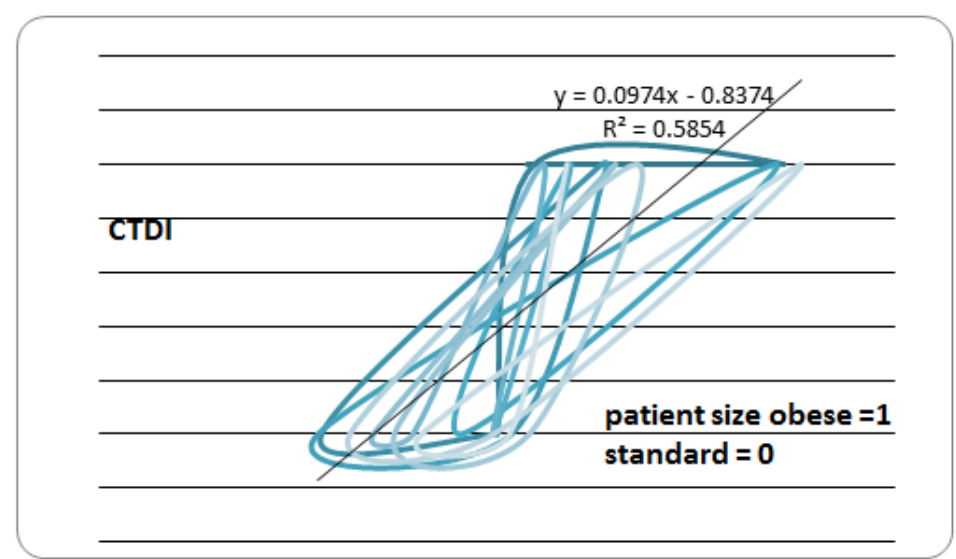

Fig-4: Correlations between patient size and CT dose index 


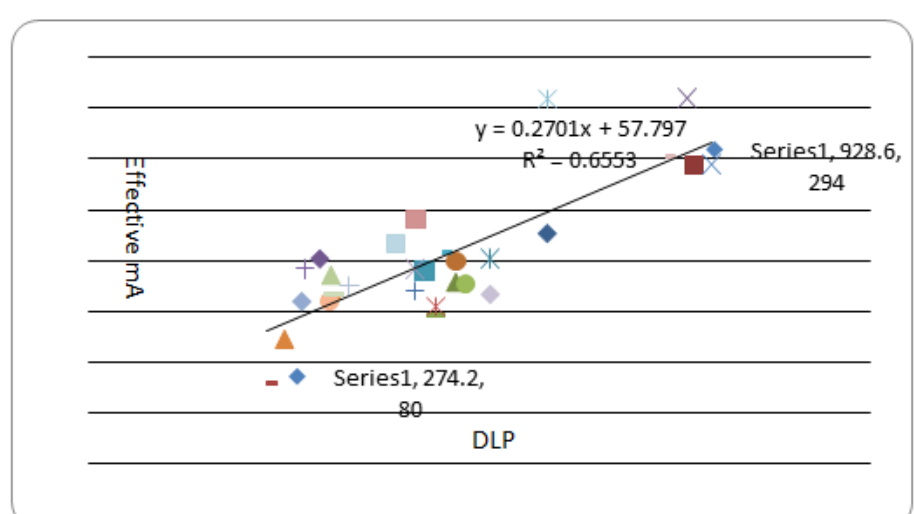

Fig-5: Correlation between effective $\mathrm{mA}$ and dose length product

\section{DISCUSSION}

The patients' characteristics (height, age weight and BMI) were differed among the sample under the study as explained in table 1. And hence the radiation dose indicated by CTDI and DLP were varying according to patients characteristics.

There was strongest and significant correlation between DLP and CTDI, where correlation coefficient $\left(\mathrm{R}^{2}\right)$ was around 0.9. So one can gathered the DLP or CTDI, if any anonymous is known, using the equation in figure 3. This result somewhat match the result reported by Zarbet al in 2010 [10], as they measured CTDI and DLP and gathered the effective dose from them for chest and abdomen for 16 slices.

Also there was correlation between effective $\mathrm{mAs}$ and DLP, as correlation Coefficient $\left(\mathrm{R}^{2}\right)$ was 0.65 . So one can gathered the effective mAs or DLP, if any anonymous is known, using the equation in figure 6 . This result was disagreed with the result revealed by linton et al in their research for pediatric CT dose [11].

Also there was correlation between CTDI and patient size in the term of weight or BMI, in which (R2) was 0.59 . This result was consistent with the result reported by Mettler et al. in 2000[12].

There was no correlation between time and dose product. Also there was no correlation between patient's ages and dose index, as the correlation coefficient were less than 0.5 .

The effective dose, which is considered radiation protection unit, during this examination could be derived from the DLP, using various published data sources [9, 15-22] and ICRP 102 [14]. As these data revealed conversion coefficient. So for this study the effective dose can be calculated by multiplying the average DLP by a factor of 0.015 for adult, and this would be equal to $8.07 \mathrm{msv}$, which is considered acceptable value in the term of radiation protection, for this type of examination.

\section{CONCLUSION AND RECOMMENDATIONS}

CTDIs can be achieved by known DLPs; Or CTDI can be achieved by known BMI using the equations shown in the results for the same machine protocol. Effective dose for abdomen CTfor the current machine protocol considered safe.

The study recommended the establishment of local diagnostic references level corresponded to international diagnostic references level available, using more scientific studies on CT doses and measurement. Also the study recommended more studies in this field specially for computed tomography angiography CTA.

\section{ACKNOWLEDGEMENT}

Researchers would thank all staff of mentioned hospitals during data collection phases, training centers and imaging department.

\section{REFERENCES}

1. American Association of Physicists in Medicine (AAPM) Report 39. Specification and Acceptance Testing of Computed Tomography Scanners. New York: AAPM, 1993.

2. McNitt-Gray MF. "AAPM/RSNA physics tutorial for residents: Topics in CT. Radiation dose in CT."Radiographics.2002; 22:1541-1553.

3. Leitz W, Axelsson B, Szendro G. "Computed tomography dose assessment: A practical approach.” RadiatProtDosim.1995; 57:377-380.

4. Boone JM. "The trouble with CTDI 100." Med Phys.2007; 34:1364-1371.

5. Mori S, Endo M, Nishizawa K, Tsunoo T, Aoyama T, Fujiwara H, Murase K. "Enlarged longitudinal dose profiles in cone-beam CT and the need for modified dosimetry." Med Phys.2005; 32:1061-1069.

6. Dixon RL. "A new look at CT dose measurement: Beyond CTDI." Med Phys.2003; 30:1272-1280.

7. A.A. Qurashi, L. Rainford, S. Foley; Dublin/IE. Establishment of diagnostic reference levels for CT trunk examinations in Saudi Arabia. file://C:/Users/mu/Downloads/ECR2014_C2256.pdf. 
8. Huda W, Ravenel JG, Scalzetti EM. "How do radiographic techniques affect image quality and patient doses in CT?" Semin Ultrasound CT MR.2002; 23:411-422.

9. International Commission on Radiological Protection (ICRP) Report 60. 1990 Recommendations of the International Commission on Radiological Protection. Annals of the ICRP 21:1/3, 1991 .

10. McCollough $\mathrm{CH}$, Schueler BA. "Calculation of effective dose." Med Phys.2000; 27:828-837.

11. Zarb F, Rainford L, McEntee MF. AP diameter shows the strongest correlation with CTDI and DLP in abdominal and chest CT. Radiation protection dosimetry. 2010 Aug 1;140(3):266-73.

12. Linton OW, Mettler Jr FA. National conference on dose reduction in $\mathrm{CT}$, with an emphasis on pediatric patients. American Journal of Roentgenology. 2003 Aug;181(2):321-9.

13. Mettler FA, Jr., Wiest PW, Locken JA, Kelsey CA. "CT scanning: Patterns of use and dose." J Radiol Prot.2000; 20:353-359.

14. Task Group on Control of Radiation Dose in Computed Tomography. Managing patient dose in computed tomography. A report of the International Commission on Radiological Protection. Annals of the ICRP. 2000;30(4):7.

15. International Commission on Radiological Protection. Managing patient dose in multidetector computed tomography (MDCT). ICRP publication 102. Elsevier, Amsterdam: ICRP; 2007.
16. Kalra MK, Maher MM, Toth TL, Schmidt B, Westerman BL, Morgan HT, Saini S. Techniques and applications of automatic tube current modulation for CT. Radiology. 2004 Dec;233(3):649-57.

17. Nishizawa K, Mori SI, Ohno M, Akahane K, Kos, Yanagawa N, Yoshida T, Wada SI, Iwai K. Patient dose estimation on multi detector-row CT from abdomen for adult and abdomen-pelvis for child examinations. Japanese Journal of Medical Physics (Igakubutsuri). 2008 Mar 31;27(4):153-62.

18. Groves AM, Owen KE, Courtney HM, Yates SJ, Goldstone KE, Blake GM, Dixon AK. 16-detector multislice CT: dosimetry estimation by TLD measurement compared with Monte Carlo simulation. The British journal of radiology. 2004 Aug;77(920):662-5.

19. Osman H, Elzaki A, Suleiman A, Sam AK. Evaluation of staff and ambient exposures during orthopedic procedures. Asian J. Med. Clin. Sci. 2012;1(1):14-8.

20. Osman H, Elzaki A, Sherif K, Sulieman A. Orthopedist's hands radiation doses during orthopedic surgery procedures. Scholars Journal of Applied Medical Sciences. 2013;1(5):369-71.

21. Osman H, Sulieman A, Sam AK. Orthopedist's thyroid radiation dose during surgery. Journal of Advanced Medical Research. 2011;1:55-60.

22. Osman H, Elzaki A, Abd Elgyoum A, Abd Elrahim E. Evaluation of Radiation Entrance Skin Dose for Pediatrics Chest X-Ray Examinations in Taif. Wulfenia Journal. 2014;21:60-7. 